\title{
Online Parameter Identification of the Lithium-Ion Battery with Refined Instrumental Variable Estimation
}

\author{
An Wen, ${ }^{1}$ Jinhao Meng $\mathbb{D}^{2},{ }^{\text {Jichang Peng, }}{ }^{3}$ Lei Cai, ${ }^{4}$ and Qian Xiao ${ }^{5}$ \\ ${ }^{1}$ School of Automation, Foshan University, Foshan 528000, China \\ ${ }^{2}$ College of Electrical Engineering, Sichuan University, Chengdu 610065, China \\ ${ }^{3}$ Smart Grid Research Institute, Nanjing Institute of Technology, Nanjing 211167, China \\ ${ }^{4}$ Faculty of Computer Science and Engineering, Xi'an University of Technology, Xi'an 710048, China \\ ${ }^{5}$ Key Laboratory of Smart Grid of Ministry of Education, Tianjin University, Nankai, Tianjin 300072, China \\ Correspondence should be addressed to Jinhao Meng; scmjh2008@163.com
}

Received 12 August 2020; Revised 9 September 2020; Accepted 20 September 2020; Published 1 October 2020

Academic Editor: Jing $\mathrm{Na}$

Copyright ( $\odot 2020$ An Wen et al. This is an open access article distributed under the Creative Commons Attribution License, which permits unrestricted use, distribution, and reproduction in any medium, provided the original work is properly cited.

\begin{abstract}
Refined Instrumental Variable (RIV) estimation is applied to online identify the parameters of the Equivalent Circuit Model (ECM) for Lithium-ion (Li-ion) battery in this paper, which enables accurate parameters estimation with the measurement noise. Since the traditional Recursive Least Squares (RLS) estimation is extremely sensitive to the noise, the parameters in the ECM may fail to converge to their true values under the measurement noise. The RIV estimation is implemented in a bootstrap form, which alternates between the estimation in the system model and the noise model. The Box-Jenkins model of the Li-ion battery transformed from the two RC ECM is selected as the transfer function model for the RIV estimation in this paper. The errors of the two RC ECM are independently generated by the residual of high-order Auto Regressive (AR) model estimation. With the benefit of a series of auxiliary models, the data filtering technology can prefilter the measurement and increase the robustness of the parameters against the noise. Reasonable parameters are possible to be obtained regardless of the noise in the measurement by RIV. Simulation and experimental tests on a LiFePO $\mathrm{Pattery}_{4}$ validate the efficiency of RIV for parameter online identification compared with traditional RLS.
\end{abstract}

\section{Introduction}

With the continuous decline of the price and the superior performance in the energy density, lithium-ion (Li-ion) battery has become an optimal choice for both the battery pack in the electric vehicle (EV) and the stationary energy storage systems in the grid [1-3]. In order to integrate the renewable energy into the grid, stationary energy storage system is critical for the inflexibility of balance and the intermittent power supply. Like the power sources, the performance of Li-ion battery also affects the acceptance of the EV produced in the market [4-8]. Especially for the pure battery-powered EV, the power capability and the energy capacity of the battery pack are closely related to the drivers' experience. Battery Management System (BMS) is in charge of the cell operation in the battery pack, which should ensure the reliability and safety of the whole pack [9-12]. The battery's states (State of Charge (SOC), State of Health $(\mathrm{SOH})$, State of Function (SOF), etc) reflect the current status of each cell in the battery pack, which must be carefully calculated from the measurement in BMS [13-16]. Although model-based estimation seems to be a practical solution for estimating the battery states, the accuracy of the estimation is closely related to the battery model from prior knowledge [17-19]. The reliability and accuracy of the battery model depend on the choice of the parameters. Moreover, some parameters can directly reflect the battery states; for example, the internal resistance and the capacity are always treated as the effective indictors to the information of the battery $\mathrm{SOH}$ [20-23].

Due to the electrochemical reactions inside the battery during the charging and discharging process, the parameters in the battery ECM are always changing with temperature and SOC [24-27]. Constant parameters of ECM in the 
battery operating process definitely reduce its accuracy. Look-up table is a possible way to improve the feasibility of the ECM under various conditions, but huge efforts are needed to build the look-up table considering the effect of temperature and SOC. Another factor should not be ignored here is that the batteries aging during their operation, which means the constant loop-up table of the parameters from the new cell may fail to accurately predict the characteristic of an old cell. The cells are connected in parallel and series into the battery pack for providing enough energy to the applications. The inconsistency of the cell leads to more workload on modelling if accurate model of each cell in the battery pack is needed $[28,29]$. Therefore, updating the parameters online is necessary for modelling accuracy and states estimation [30, 31]. Recursive Least Squares (RLS) and Kalman filter are generally applied to identify the parameters of ECM [32-36]. RLS easily fails to converge to the actual value of the parameters when the measurement contains noise [11]. Kalman filter related methods usually have a higher computational complexity than RLS. As explained in [37], the system order of the Kalman filter-related methods is usually high, which makes it difficult to tune the algorithm. Extended Kalman filter (EKF) is applied to identify the parameters of the two RC ECM, $7 \times 7$ order matrix is calculated and updated in each step for parameters identification [38]. The parameters of the battery ECM with one RC are estimated by the moving window least square method with AutoRegressive (AR) model, but the effect of the window width on the accuracy of identified parameter have not been discussed [35]. Algorithms with more computing complexity, such as, Sigmapoint Kalman filter [39] and H-infinity filter [31] are also used to online update the parameters of the battery model. Furthermore, the heuristic algorithms including Genetic Algorithm (GA) [40] and Particle Swarm Optimization (PSO) [41] with a moving window are applied to obtain the parameters in ECM. However, fewer efforts have focused on dealing with the effect of the measurement noise on the identified parameters in ECM. The noise from sensors has always existed during the measurement of the battery current and voltage, which may influence the convergence of the estimated parameters to the true values in real applications. Therefore, online parameter identification algorithm with good enough ability to obtain reasonable parameters under measurement noise conditions is still needed.

In order to identify reasonable parameters of the battery ECM considering the effect of the measurement noise, the Refined Instrumental Variable (RIV) estimation is firstly applied to identify the parameters of the two RC ECM online in this paper. RIV focuses on improving the performance of RLS with the data-filtering technology in a bootstrap form. The Box-Jenkins model containing the battery system model and the AutoRegressive Moving Average (ARMA) noise model is established for the parameter identification in this paper. The parameters of the ARMA noise model are estimated from the auxiliary battery model with parameters in the previous step. High-order AR model independently generates the estimation of the input in the noise model. The data filtering technology helps the RIV estimation to obtain reasonable parameters with measurement noise.
The reminder of this paper is as following. The BoxJenkins battery model is described in Section 2. The details of RIV for parameter identification is introduced in Section 3. The simulation results of the proposed method are shown in Section 4 . The RIV estimation is experimentally validated on the measurements from a $\mathrm{LiFePO}_{4}$ battery in Section 5 . The conclusions are given in Section 6.

\section{Box-Jenkins Model of Lithium-Ion Battery}

In order to identify the parameters of the battery model online with RIV, a suitable battery transfer function has to be established at first. Describing the output error of the battery model as an AMRA model, the form of the Box-Jenkins model is expressed as the following form [42]:

$$
y(k)=\frac{B\left(z^{-1}\right)}{A\left(z^{-1}\right)} u(k)+\frac{D\left(z^{-1}\right)}{C\left(z^{-1}\right)} e(k),
$$

where $e(k)$ is the white noise with zero mean and variance $\sigma^{2}$ and $u(k)$ and $y(k)$ are the input and output of the system. If the noise-free output of the system is defined as $x(\mathrm{k})$, the Box-Jenkins model of equation (1) can be decomposed into equations (2)-(4). Among them, equation (3) can be seen as the system model and equation (4) is the noise model.

$$
\begin{aligned}
& y(k)=x(k)+v(k), \\
& x(k)=\frac{B\left(z^{-1}\right)}{A\left(z^{-1}\right)} u(k), \\
& v(k)=\frac{D\left(z^{-1}\right)}{C\left(z^{-1}\right)} e(k) .
\end{aligned}
$$

In the above equations, the terms $A\left(\mathrm{z}^{-1}\right), B\left(\mathrm{z}^{-1}\right), C\left(\mathrm{z}^{-1}\right)$, and $D\left(\mathrm{z}^{-1}\right)$ are normally defined as follows:

$$
\begin{aligned}
& A\left(z^{-1}\right)=1+a_{1} \cdot z^{-1}+a_{2} \cdot z^{-2}+\cdots+a_{n_{a}} \cdot z^{-n_{a}}, \\
& B\left(z^{-1}\right)=b_{0}+b_{1} \cdot z^{-1}+b_{2} \cdot z^{-2}+\cdots+b_{n_{b}} \cdot z^{-n_{b}}, \\
& C\left(z^{-1}\right)=1+c_{1} \cdot z^{-1}+c_{2} \cdot z^{-2}+\cdots+c_{n_{c}} \cdot z^{-n_{c}}, \\
& D\left(z^{-1}\right)=1+d_{1} \cdot z^{-1}+d_{2} \cdot z^{-2}+\cdots+d_{n_{d}} \cdot z^{-n_{d}} .
\end{aligned}
$$

It can be seen from the structure of the Box-Jenkins model that the transfer function of the system model and the noise model are two independently parametrized rational functions. The optimal prefilter for all the related measurement in the battery model is derived from the noise model in the Box-Jenkin model.

One RC ECM is easy for parameter calculation and is more stable to uncertainties $[43,44]$. Compared with one RC model, one more RC network is used so that the fast dynamic and short dynamic of the battery terminal voltage can be described, respectively. Of course, more calculation is needed for two RC ECM. Two RC ECM is popular in Li-ion battery modelling area because it is a good trade-off between accuracy and complexity [45]. Thus, we choose two RC ECM to show the advantages of the methods in this paper. It 
should be noted that the method in this work is also suitable for one RC ECM. The structure of the two RC ECM is illustrated in Figure 1.

The two RC model consists of resistance, capacitance, and voltage source to represent the battery characteristics. The voltage source stands for the OCV-SOC relationship, and $R_{0}$ is the internal resistance. The expression of the two RC ECM in Figure 1 is shown as follows:

$$
\left\{\begin{array}{l}
\dot{U}_{1}=-\frac{U_{1}}{R_{1} \cdot C_{1}}+\frac{I_{L}}{C_{1}} \\
\dot{U}_{2}=-\frac{U_{2}}{R_{2} \cdot C_{2}}+\frac{I_{L}}{C_{2}} \\
U_{t}=U_{o c}-U_{1}-U_{2}-I_{L} \cdot R_{0}
\end{array}\right.
$$

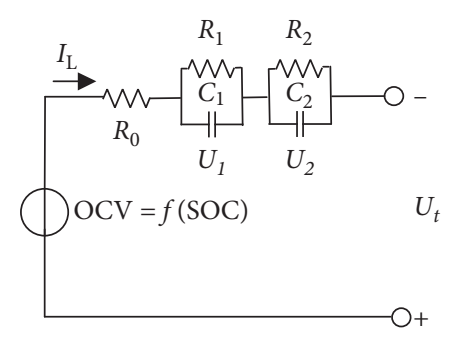

Figure 1: The structure of two RC ECM.

According to equation (6), the discretization form of the two RC ECM is as follows:

$$
\left\{\begin{array}{l}
U_{t}(k)=f(\mathrm{SOC})-I_{L}(k) \cdot R_{0}-U_{1}(k)-U_{2}(k), \\
U_{1}(k)=\exp \left(\frac{-T_{s}}{\tau_{1}}\right) \cdot U_{1}(k-1)+R_{1} \cdot\left(1-\exp \left(\frac{-T_{s}}{\tau_{1}}\right)\right) \cdot I_{L}(k-1), \\
U_{2}(k)=\exp \left(\frac{-T_{s}}{\tau_{2}}\right) \cdot U_{2}(k-1)+R_{2} \cdot\left(1-\exp \left(\frac{-T_{s}}{\tau_{2}}\right)\right) \cdot I_{L}(k-1),
\end{array}\right.
$$

where $T_{\mathrm{s}}$ is the sampling time, $\tau_{1}$ and $\tau_{2}$ are the two time constants of the RC networks in the ECM.

Afterwards, let us define $z$ as the shift operator. Equation (7) can be transformed into the following form:

$$
x_{0}(k)=\frac{B\left(z^{-1}\right)}{A\left(z^{-1}\right)} \cdot I_{L}(k)=\frac{b_{0}+b_{1} \cdot z^{-1}+b_{2} \cdot z^{-2}}{1+a_{1} \cdot z^{-1}+a_{2} \cdot z^{-2}} \cdot I_{L}(k) \text {, }
$$

where $x_{o}(k)$ is the deviation of the terminal voltage $U_{\mathrm{t}}$ and the open circuit voltage $f$ (SOC). The coefficients $a_{1}, a_{2}, b_{0}$, $b_{1}$, and $b_{2}$ in equation (8) are expressed as follows:

$$
\left\{\begin{array}{l}
a_{1}=-\left(\exp \left(\frac{-T_{s}}{\tau_{1}}\right)+\exp \left(\frac{-T_{s}}{\tau_{2}}\right)\right) \\
a_{2}=\exp \left(\left(\frac{-T_{s}}{\tau_{1}}\right)+\left(\frac{-T_{s}}{\tau_{2}}\right)\right) \\
b_{0}=R_{0} \\
b_{1}=\left(R_{0} \cdot\left(-\exp \left(\frac{-T_{s}}{\tau_{1}}\right)-\exp \left(\frac{-T_{s}}{\tau_{2}}\right)\right)+R_{1} \cdot\left(1-\exp \left(\frac{-T_{s}}{\tau_{1}}\right)\right)+R_{2} \cdot\left(1-\exp \left(\frac{-T_{s}}{\tau_{2}}\right)\right)\right) \\
b_{2}=\left(R_{0} \cdot\left(\exp \left(\left(\frac{-T_{s}}{\tau_{1}}\right)+\left(\frac{-T_{s}}{\tau_{2}}\right)\right)\right)+R_{1} \cdot \exp \left(\frac{-T_{s}}{\tau_{2}}\right) \cdot\left(\exp \left(\frac{-T_{s}}{\tau_{1}}\right)-1\right)\right)
\end{array}\right.
$$


The noise model is selected in AMRA form with oneorder AR model and MA model, which can be expressed as follows:

$$
v(k)=\frac{1+d_{1} \cdot z^{-1}}{1+c_{1} \cdot z^{-1}} e(k) .
$$

According to the battery model in equation (8) and the noise model in equation (10), the Box-Jenkins model of the two RC ECM is

$$
\begin{aligned}
y(k)= & u_{o}(k)+v(k)=\frac{b_{0}+b_{1} \cdot z^{-1}+b_{2} \cdot z^{-2}}{1+a_{1} \cdot z^{-1}+a_{2} \cdot z^{-2}} \cdot I_{L}(k) \\
& +\frac{1+d_{1} \cdot z^{-1}}{1+c_{1} \cdot z^{-1}} e(k),
\end{aligned}
$$

where the parameters in equation (11) are possible to be identified by RIV, and the noise model can be used as the prefilter for improving the robustness of RIV against the measurement noise.

\section{RIV Estimation}

RIV proposed by $[46,47]$ has particular advantage in dealing with the noise in the measurement. Moreover, RIV has provided a practical solution to the maximum likelihood function for the Box-Jenkins model [42] and is able to obtain more reliable results for the stiff dynamic system than the prediction error minimization method [47]. The structure of the RIV estimation is shown in Figure 2. It mainly consists of two estimation processes: the parameters in the battery model and the parameters in the noise model.

The noise model needs to be identified before prefiltering all the variables related to the battery model. The error function of the noise model is defined as

$$
w(k)=\frac{C\left(z^{-1}\right)}{D\left(z^{-1}\right)} \cdot v(k)-e(k) .
$$

As described in Figure 2, the prefilter of the noise model is

$$
f_{2}\left(z^{-1}\right)=\frac{1}{D\left(z^{-1}\right)} .
$$

Substitute the prefilter $f_{2}\left(z^{-1}\right)$ into the error function $w$ (k); the following equation is obtained:

$$
w(k)=C\left(z^{-1}\right) \cdot v_{f_{2}}(k)-D\left(z^{-1}\right) \cdot e_{f 2}(k)
$$

where the $v_{f_{2}}(k)$ and $e_{f 2}(k)$ are the prefiltered variables.

However, $v(k)$ and $e(k)$ are not known in the beginning of the parameter identification, and the profiler $f_{2}\left(z^{-1}\right)$ is also hardly known in advance. Auxiliary models are needed to calculate these variables in this condition. The instrumental variable $\widehat{v}(k)$ is obtained as

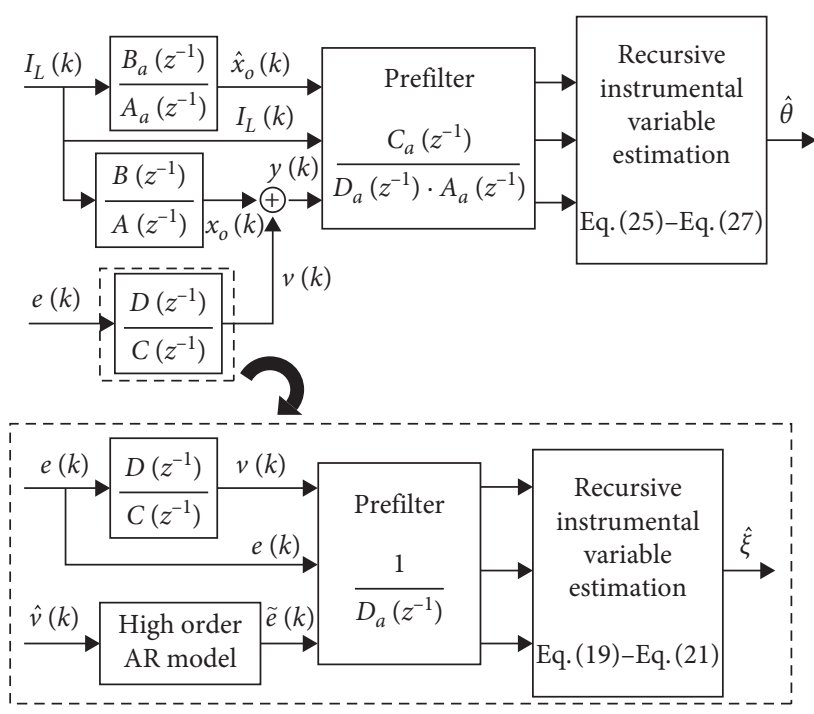

FIgURE 2: The diagram of the RIV estimation.

$$
\widehat{v}(k)=y(k)-\widehat{x}(k),
$$

where $\hat{x}(k)$ is calculated from the auxiliary battery model $\left(B_{a}\left(z^{-1}\right) / A_{a}\left(z^{-1}\right)\right)$.

Then, $\tilde{e}(k)$ can be independently calculated from the residuals of the high-order AR model of $v(k)$. The unknown variables in equation (11) are able to be replaced by $\widetilde{e}(k)$ and $\widehat{v}(k)$. Afterwards, the parameters in the noise model are able to be updated as the following recursive process:

$$
\widehat{\xi}(k)=\widehat{\xi}(k-1)+L_{e}(k) \cdot\left(\tilde{e}_{f 2}(k)-\psi^{T}(k) \cdot \widehat{\xi}(k-1)\right),
$$

$L_{e}(k)=\widehat{P}_{e}(k-1) \cdot \widehat{\psi}(k) \cdot\left[\sigma^{2}+\psi^{T}(k) \cdot \widehat{P}_{e}(k-1) \cdot \widehat{\psi}(k)\right]^{-1}$,

$$
\widehat{P}_{a}(k)=\widehat{P}_{a}(k-1)-L_{a}(k) \cdot \varphi^{T}(k) \cdot \widehat{P}_{a}(k-1),
$$
where $\xi(k)=\left[\begin{array}{lll}1 & c_{1} & d_{1}\end{array}\right]^{T}$,

$$
\begin{aligned}
& \psi(k)=\left[\begin{array}{lll}
\widehat{v}_{f 2}(k) & \widehat{v}_{f 2}(k-1) & -\widetilde{e}_{f 2}(k-1)
\end{array}\right]^{T}, \\
& \widehat{\psi}(k)=\left[\begin{array}{lll}
\widehat{v}_{f 2}(k) & \widehat{v}_{f 2}(k-1) & -\widehat{e}_{f 2}(k-1)
\end{array}\right]^{T} .
\end{aligned}
$$

The prefiltered output $\widehat{e}_{f 2}(k)$ is calculated from the auxiliary ARMA noise model as follows:

$$
\widehat{e}_{f 2}(k)=\frac{C_{a}\left(z^{-1}\right)}{D_{a}\left(z^{-1}\right)} \cdot \widehat{v}_{f 2}(k),
$$

where the parameters in $C_{a}\left(z^{-1}\right)$ and $D_{a}\left(z^{-1}\right)$ are obtained from the parameters in the previous step.

After calculating the parameters in the noise model, the prefilter $f_{1}\left(z^{-1}\right)$ can be used for the variables in the battery model. The expression of the prefilter $f_{1}\left(z^{-1}\right)$ is as follows:

$$
f_{1}\left(z^{-1}\right)=\frac{C_{a}\left(z^{-1}\right)}{D_{a}\left(z^{-1}\right) \cdot A_{a}\left(z^{-1}\right)}
$$


Since the noise-free output is still unknown, the following auxiliary battery model is used to solve this problem.

$$
\widehat{x}_{0}(k)=\frac{B_{a}\left(z^{-1}\right)}{A_{a}\left(z^{-1}\right)} \cdot I_{L}(k),
$$

where the parameters in the auxiliary battery model come from the estimation results of the previous step.

After the measurement prefiltering process, the parameters in the battery model are updated as the following equations:

$$
\hat{\theta}(k)=\hat{\theta}(k-1)+L_{a}(k) \cdot\left(y_{f 1}(k)-\varphi^{T}(k) \cdot \hat{\theta}(k-1)\right),
$$

$$
\begin{gathered}
L_{a}(k)=\widehat{P}_{a}(k-1) \cdot \widehat{\varphi}(k) \cdot\left[\sigma^{2}+\varphi^{T}(k) \cdot \widehat{P}_{a}(k-1) \cdot \widehat{\varphi}(k)\right]^{-1}, \\
\widehat{P}_{a}(k)=\widehat{P}_{a}(k-1)-L_{a}(k) \cdot \varphi^{T}(k) \cdot \widehat{P}_{a}(k-1),
\end{gathered}
$$

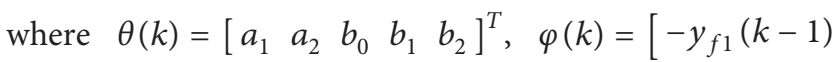
$\left.-y_{f 1}(k-2)-I_{L f 1}(k)-I_{L f 1}(k-1)-I_{L f 1}(k-2)\right]^{T}$, $\widehat{\varphi}(k)=\left[-\widehat{x}_{o f 1}(k-1)-\widehat{x}_{o f 1}(k-2)-I_{L f 1}(k)-I_{L f 1}(k-1)\right.$ $\left.-I_{L f 1}(k-2)\right]^{T}$.

According to the previous steps, the RIV is able to identify the parameters of the battery model with measurement noise. The main steps of the RIV estimation are summarized as follows:

Step 1. Parameters identification of the ARMA noise model: Prefiltering $v(k)$ and $e(k)$ forms $v_{f_{2}}(k)$ and $e_{f_{2}}(k)$. Using auxiliary noise model (equation (19)) to calculate $\hat{e}(k)$ and equation (15) for calculating $\widehat{v}_{f 2}(k)$. High-order AR model of $v(k)$ is selected to obtain $\widetilde{e}_{f 2}(k)$. Equations (16)-(18) are then applied to estimate the parameters in the ARMA noise model.

Step 2. Parameters identification of the battery model: Prefiltering $y(\mathrm{k}), x_{o}(k)$ and $I_{L}(k)$ to receive the variables $y_{f_{1}}(k), x_{\text {of } 1}(k)$, and $I_{L f 1}(k)$. The auxiliary battery model equation (22) is used to calculate $\hat{x}_{\text {of } 1}(k)$. Afterwards, equations (23)-(25) are used to estimate the parameters in the battery model.

Step 3. Update the covariance matrix: The covariance matrices $\widehat{P}_{a}$ and $\widehat{P}_{e}$ can be updated according to the new input $\hat{\varphi}(k)$ and $\widehat{\psi}(k), \quad \widehat{P}_{a}=\sigma^{2}$. $\left[\sum_{i=1}^{N} \widehat{\varphi}(k) \cdot \widehat{\varphi}^{T}(k)\right]^{-1}, \widehat{P}_{e}=\sigma^{2} \cdot\left[\sum_{i=1}^{N} \widehat{\psi}(k) \cdot \widehat{\psi}^{T}(k)\right]^{-1}$.

\section{Simulation Validation}

Current pulse profile is used in simulation to validate the performance of the parameter identification method in this paper. More specifically, the current pulse is applied to the ECM in simulation for verifying the advantage of RIV in parameters identification under measurement noise condition. RIV is compared with traditional RLS to show its improvement in parameter identification.
For simplifying the parameter identification process, the voltage source in the ECM is omitted. The equivalent circuit in Figure 1 is used for the validation of the parameter identification method. The true values of the parameters in simulation are listed in Table 1. The two-time constants of the RC networks are different from each other, which represent the fast dynamic and slow dynamic, respectively.

White Gaussian noises with specific variances are added to the battery terminal voltage in simulation. The standard deviations of the noise are set to $1 \mathrm{mV}$ and $10 \mathrm{mV}$, respectively, in this paper [48]. In order to evaluate the performance of the parameter identification method, Monte Carlo simulations are carried out for 10 times. The initial values of all the parameters are chosen to be zero and the initial covariance matrix is set to $110^{5}$ for both RIV and RLS. The order of noise model in the Box-Jenkins model is set to 1. As seen from the Bode plots in Figure 3, the identified results from traditional RLS have clearly bias error in Figure 3(a). The deviation becomes larger in all the frequencies when large standard deviations of noises are added to the measurement. Even for the lower noise level, the identified results are still obviously biased from the true system for RLS. As shown from the results in Figure 3(a), traditional RLS is quite sensitive to measurement noise. On the contrary, the results of RIV in Figure 3(b) stay close to the true transfer function in all frequencies regardless of the measurement noise, which proves the validation of the parameter identification method in this paper.

The simulation results of the traditional RLS and the RIV are shown in Table 2. The estimation results from RIV clearly converge to the true values in the simulation, while the estimated parameters in RLS converge to the wrong values. It is clearly seen from the results with the $10 \mathrm{mV}$ standard deviation of the measurement noise. The estimated parameters are further transformed into the RC parameters in Table 3. As shown in Table 3, the RC parameters from RIV are much closer to the reference than RLS.

\section{Experimental Test}

To further validate the parameter identification method in the real battery, the measurement from $\mathrm{LiFePO}_{4}$ battery is applied to the RIV estimation. As shown in Figure 4, the experimental tests are carried out on a battery test bench including a host computer, a MACCOR 4000 series test station, and a $\mathrm{LiFePO}_{4}$ battery. The specific current is used to charge and discharge the battery in the test chamber. The measurement data is sent back to the host computer for further analysis. The accuracy is $\pm 0.01 \%+1$ digit for the voltage measurement and $\pm 0.02 \%+1$ digit for the current measurement. The nominal capacity of the $\mathrm{LiFePO}_{4}$ battery used in this paper is $10 \mathrm{Ah}$, and the range of the battery voltage is between $2.0 \mathrm{~V}$ and $3.65 \mathrm{~V}$.

In order to validate the online parameter identification method in this paper, New European Driving Cycles (NEDC) is used to discharge the $\mathrm{LiFePO}_{4}$ battery. The ambient temperature is set to $25^{\circ} \mathrm{C}$ in the test chamber and the sample time is 1 second for all the tests. A scaled multiple NEDC driving cycle is used to discharge the battery to its 
TABle 1: Parameters of the battery model in simulation.

\begin{tabular}{lccccccc}
\hline Parameters & $R_{0}$ & $R_{1}$ & $C_{1}$ & $R_{2}$ & $C_{2}$ & $\tau_{1}$ & $\tau_{2}$ \\
\hline Value & 0.0151 & 0.0045 & 1261 & 0.0099 & 5987 & 5.6745 & 59.2713 \\
\hline
\end{tabular}
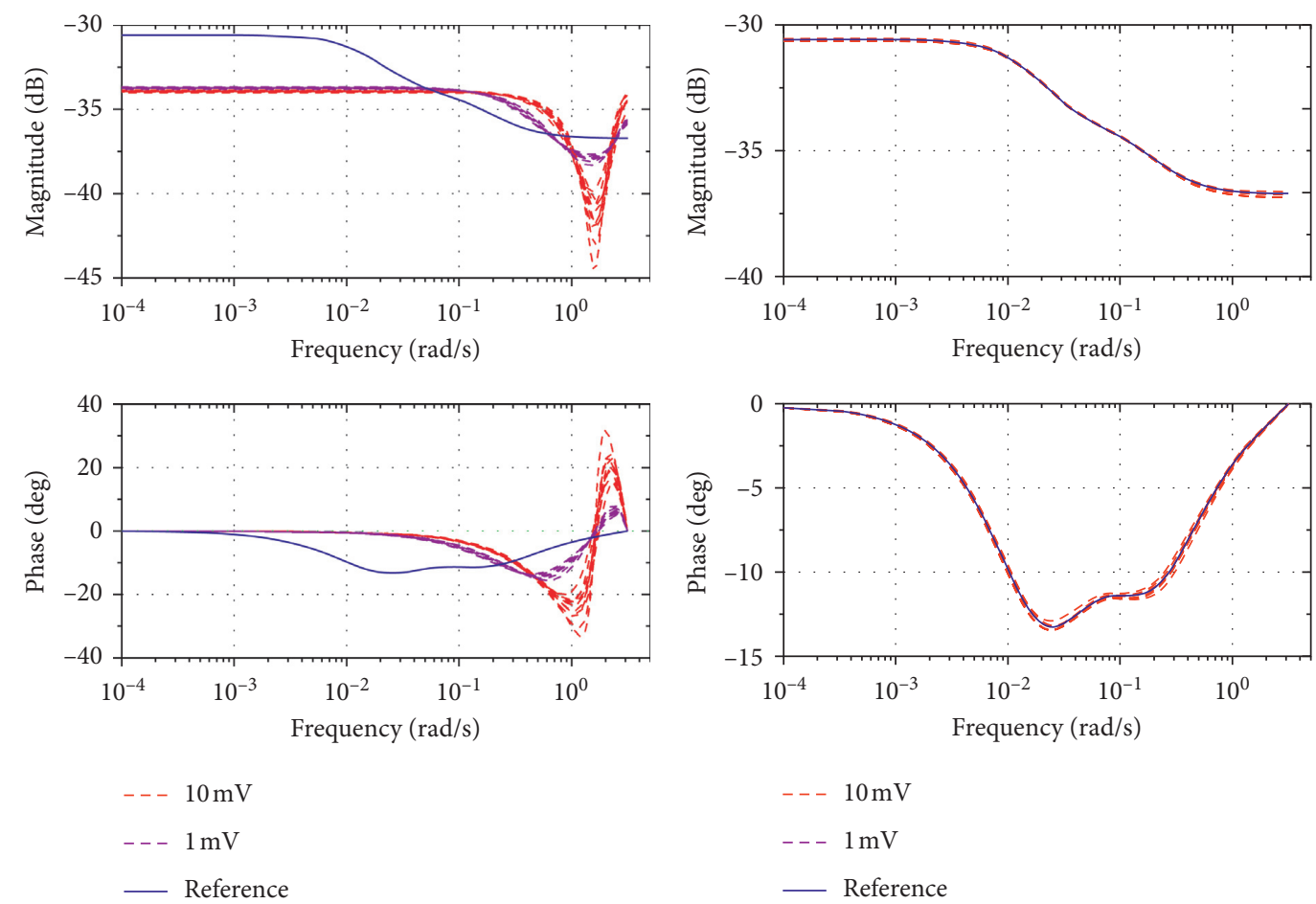

(a)

(b)

FIGURE 3: Simulation results of the parameter identification methods with different measurement noise levels. (a) Traditional RLS. (b) RIV.

TABLE 2: The direct results of the parameter identification method.

\begin{tabular}{|c|c|c|c|c|c|c|}
\hline Parameters & & $a_{0}$ & $a_{1}$ & $b_{0}$ & $b_{1}$ & $b_{2}$ \\
\hline \multirow{2}{*}{$10 \mathrm{mV}$} & RLS & 0.0057 & 0.0135 & 0.0140 & $8.2493 e-04$ & 0.0056 \\
\hline & RIV & -1.8201 & 0.8229 & 0.0151 & -0.02655 & 0.01155 \\
\hline \multirow{2}{*}{$1 \mathrm{mV}$} & RLS & -0.2201 & -0.2521 & 0.0146 & -0.0025 & -0.0013 \\
\hline & RIV & -1.8217 & 0.8244 & 0.0151 & -0.02662 & 0.01160 \\
\hline True value & & -1.822 & 0.8244 & 0.0151 & -0.02661 & 0.01159 \\
\hline
\end{tabular}

cut-off voltage. The detailed measurement during the NEDC profile is shown in Figure 5. The battery terminal voltage decreases much faster in lower SOC area. The OCV of the battery in this experiment is measured with 5\% SOC interval under charge and discharge condition, respectively. The average OCV between charge and discharge condition is used to establish the OCV-SOC relationship, as shown in Figure 6. We fit an eighth-order polynomial function via quadratic optimization by using MATLAB "quadprog" for obtaining the coefficients [49]. After constraints the diminishing OCV with the decrease of SOC, the OCV-SOC function is represented as follows:

$$
\begin{aligned}
\mathrm{OCV}= & -330.2741 \cdot \mathrm{SOC}^{8}+1507.8350 \cdot \mathrm{SOC}^{7} \\
& -2869.7023 \cdot \mathrm{SOC}^{6} \\
& +2949.8632 \cdot \mathrm{SOC}^{5}-1773.9467 \cdot \mathrm{SOC}^{4} \\
& +632.0383 \cdot \mathrm{SOC}^{3}-128.9882 \cdot \mathrm{SOC}^{2} \\
& +13.8940 \cdot \mathrm{SOC}+2.6371 .
\end{aligned}
$$

The estimated parameters of the two RC ECM during the calculating process of RLS and RIV are shown in Figures 7 and 8. Although both RLS and RIV are able to accurately estimate the battery internal resistance, RIV is possible to 
TABLE 3: The identified parameters in the two RC ECM with $0.01 \mathrm{mV}$ noise.

\begin{tabular}{lcccccrr}
\hline Parameters & $R_{0}$ & $R_{1}$ & $C_{1}$ & $R_{2}$ & $C_{2}$ & $\tau_{1}$ \\
\hline RLS & 0.0151 & 0.0011 & 1820.9 & 0.0100 & 2045.8 & 2.0100 & 20.5340 \\
RIV & 0.0151 & 0.0045 & 1260.9 & 0.0099 & 5986.7 & 5.6740 & 59.2691 \\
True value & 0.0151 & 0.0045 & 1261 & 0.0099 & 5987 & 5.6745 & 59.2713 \\
\hline
\end{tabular}

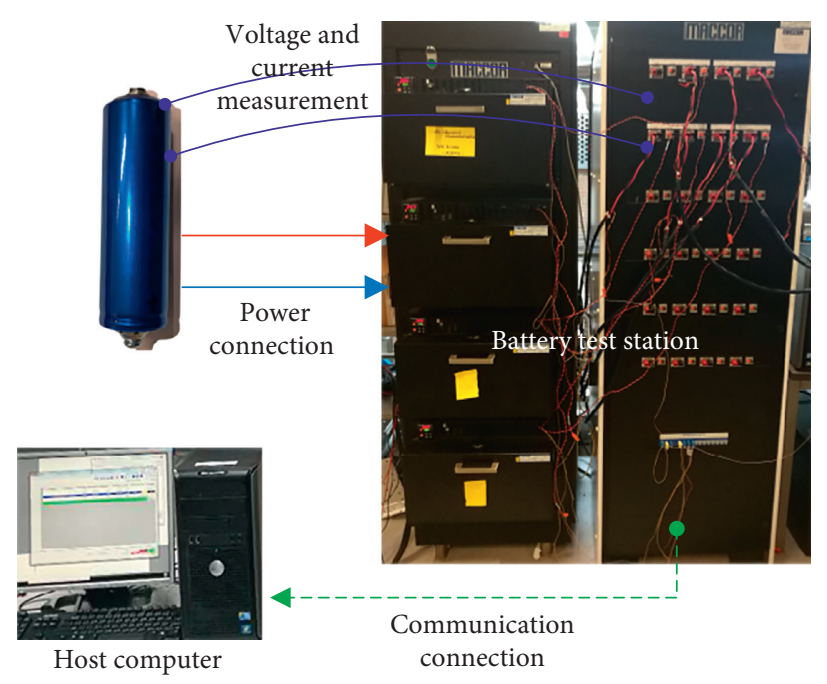

Figure 4: Structure of the test bench.

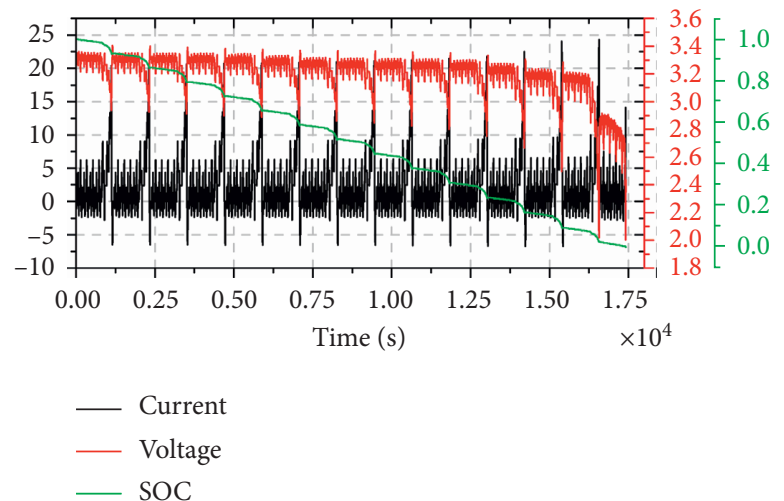

FIgURe 5: Measurement in NEDC.

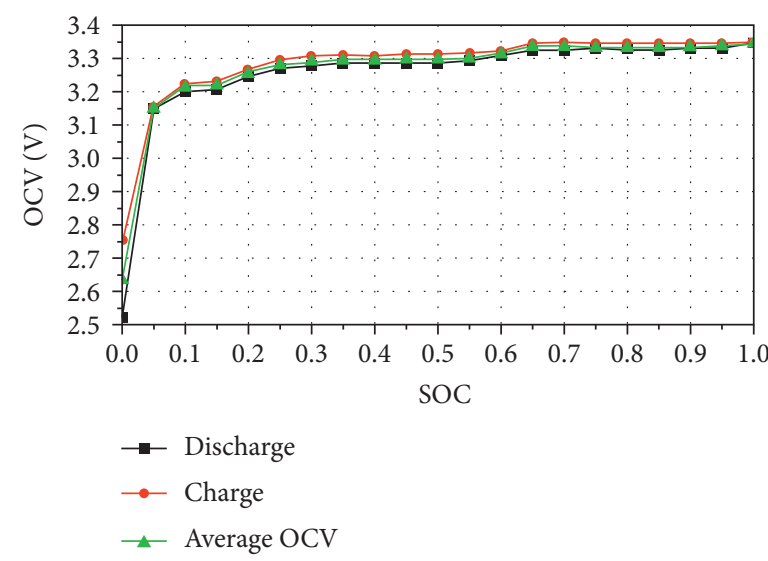

FIGURE 6: OCV-SOC relationship. 


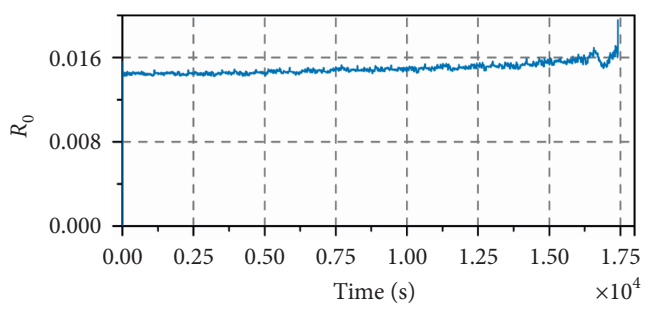

(a)

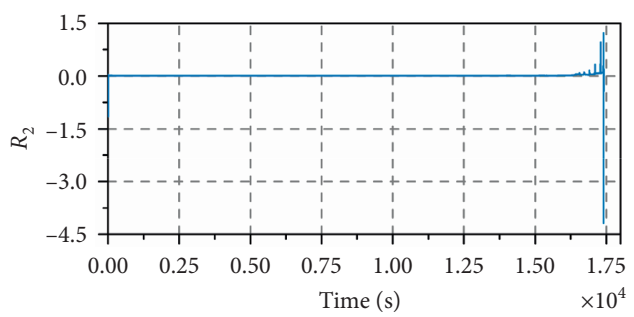

(c)

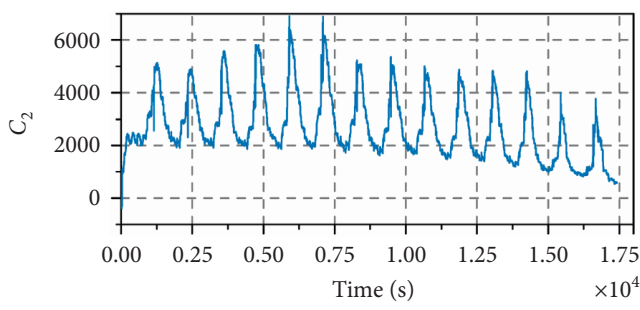

(e)

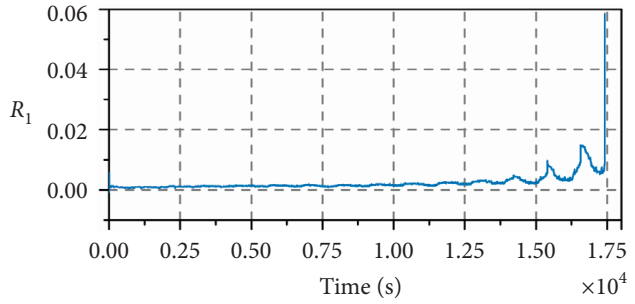

(b)

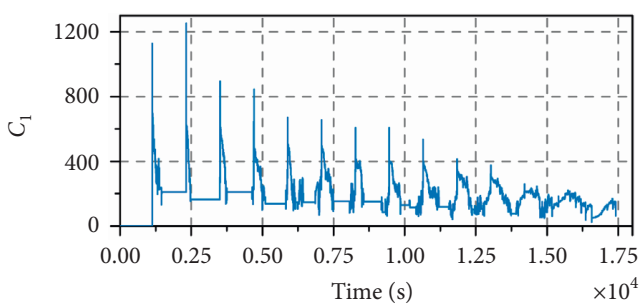

(d)

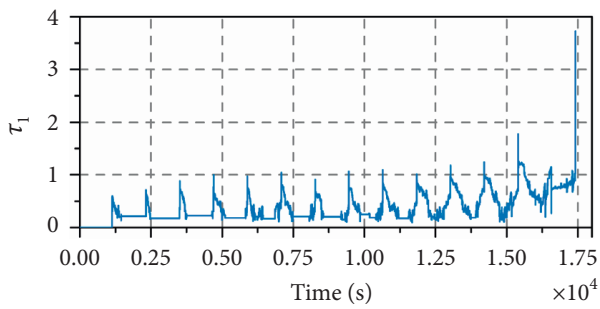

(f)

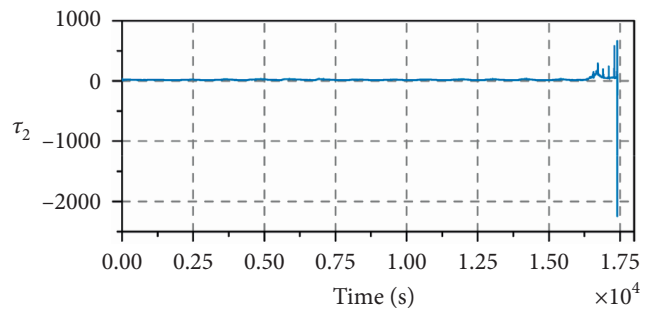

(g)

Figure 7: The identified parameters from RLS.

obtain more reasonable estimation for the rest parameters. Especially, the estimation results of $C_{1}$ and $C_{2}$ in RLS are oscillated in a wide range in Figure 8 , while the variation of the $C_{1}$ and $C_{2}$ in RIV are more reasonable as shown in Figure 8 . In order to validate the performance of the two RC ECM, the identified parameters from RLS and RIV are substituted into the two RC ECM. The prediction of the battery terminal voltage is shown in Figure 9. The terminal voltage from the two algorithms stay close to the measurement, but the details in Figure 9 show that the two RC ECM using the parameters from RIV is closer to the reference. The results are confirmed by Figure 10, which the absolute errors of the predicted terminal voltage are calculated. As shown in the enlarged figure of Figure 10, the absolute error of the terminal voltage with the estimated parameters from RIV is lower than that from RLS. In order to verify the complexity of the proposed method, the computing time on a laptop $(1.6 \mathrm{GHz} \mathrm{CPU} / 64$-bit system/ MATLAB 2019b) is calculated. After 10 times evaluation, the average computing time for each step is only $0.001 \mathrm{~s}$, which proves the good efficiency of the RIV estimation.

The mean value of the identified parameters during the discharging profile and the performance of the two RC ECM are evaluated in Table 4. The Mean Absolute Error (MAE) of the terminal voltage using parameters from RIV is $0.0219 \mathrm{~V}$, and it is $0.0287 \mathrm{~V}$ for RLS. The RMSE of the RIV is also lower than using the parameters from RLS. This is mainly because the identified time constants in the two RC ECM are more reasonable in RIV as shown in Table 4. Therefore, combined with the results from simulation in the previous section, the advantages of RIV in identifying the parameters under measurement noise are proved compared with RLS. 


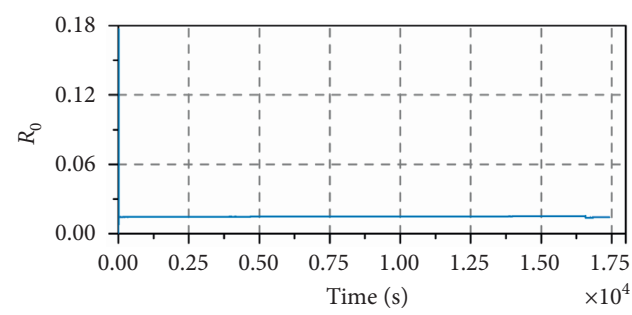

(a)

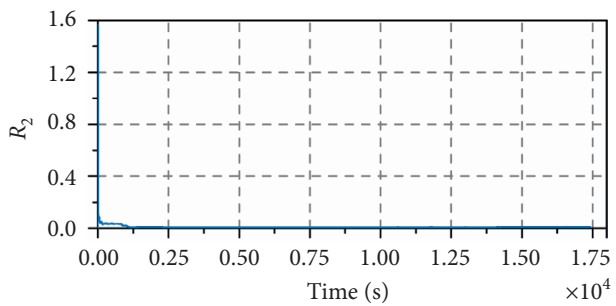

(c)

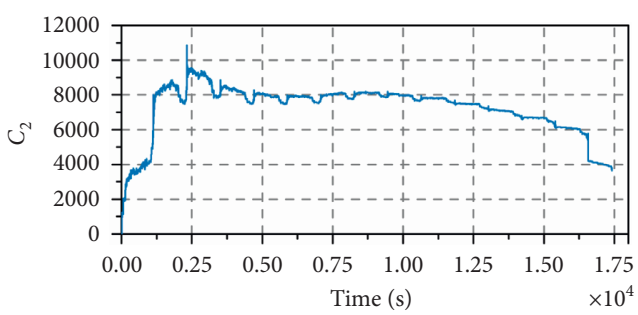

(e)

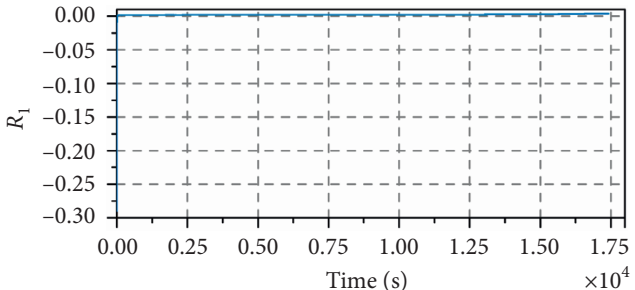

(b)

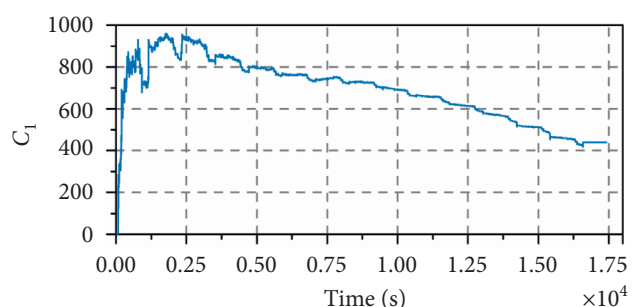

(d)

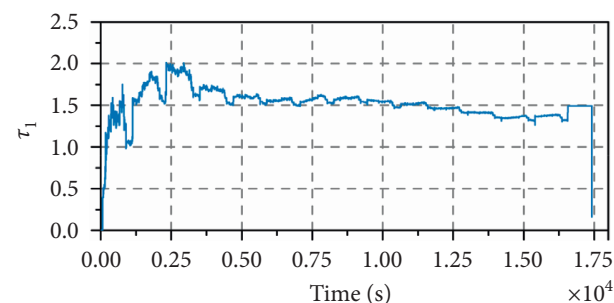

(f)

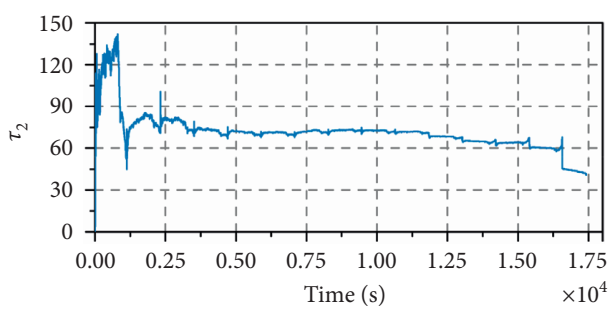

(g)

FIgURE 8: The identified parameters from RIV.

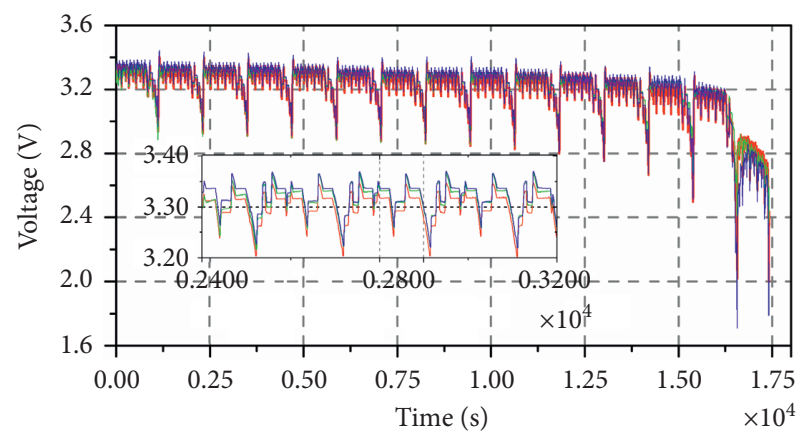

— Reference

RIV

- RLS

Figure 9: Terminal voltage prediction of the two RC ECM.

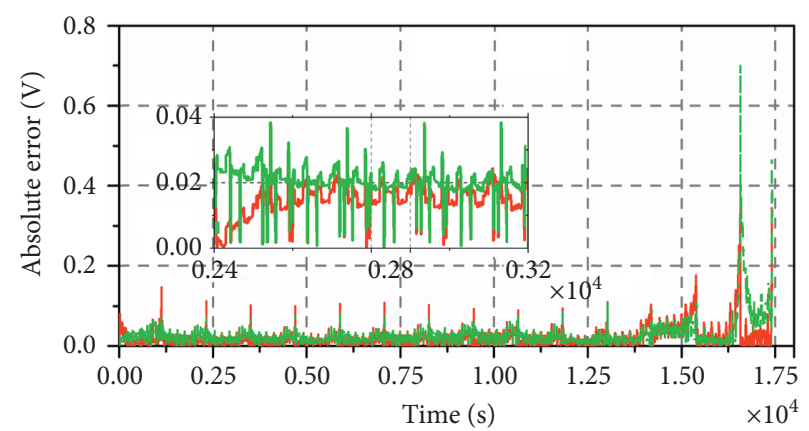

- RIV

- RLS

FIgURE 10: Absolute error of the terminal voltage prediction. 
TABLE 4: The estimation results of RLS and RIV in NEDC.

\begin{tabular}{lccccccccc}
\hline Methods & $R_{0}$ & $R_{1}$ & $R_{2}$ & $C_{1}$ & $C_{2}$ & $\tau_{1}$ & $\tau_{2}$ & MAE (V) & RMSE (V) \\
\hline RLS & 0.0149 & 0.0023 & 0.0096 & 179.0710 & 2674.4712 & 0.3682 & 20.7467 & 0.0287 & 0.0417 \\
RIV & 0.0148 & 0.0023 & 0.0117 & 687.1209 & 7224.4477 & 1.5127 & 71.5291 & 0.0219 & 0.0313 \\
\hline
\end{tabular}

\section{Conclusion}

This paper firstly uses the RIV to identify the parameters of the two RC ECM in battery modelling area, which facilities obtaining more reasonable parameters online with the disturbance in the measurement noise. Traditional RLS is sensitive to the noise in the measurement, which is possible to be solved by the data-filtering technology in the RIV. The battery BoxJenkins model for parameter identification is established at first. With the benefit of the auxiliary battery model and the auxiliary noise ARMA model, RIV is able to identify all the parameters in Box-Jenkins model separately with the prefiltered measurement. High-order AR model estimation is applied to independently generate the input of the noise model. The simulation of the equivalent circuit with different noise level proves the validation of RIV in dealing with the measurement noise. While traditional RLS fails to coverage to the true value, RIV is still able to accurately estimate the parameters in simulation. The measurement from a $\mathrm{LiFePO}_{4}$ battery further proves the validation of RIV in parameter identification. The predicted terminal voltage of the two RC ECM shows that using the identified parameters from RIV is more accurate than from RLS, because the identified time constants from RIV are more reasonable. Moreover, the variations of the parameters (Figures 7 and 8 ) in the estimation process are more appropriate for RIV compared with RLS.

Future work is needed for quantitative analysis of the measurement noise in experimental validation so that the advantages of RIV can be confirmed.

\section{Data Availability}

The battery cycling data used to support the findings of this study are currently under embargo while the research findings are commercialized. Requests for data, 12 months after publication of this article, will be considered by the corresponding author.

\section{Conflicts of Interest}

The authors declare that there are no conflicts of interest regarding the publication of this paper.

\section{Acknowledgments}

This work was supported by Foshan Innovation Fund, project no. FS0AA-KJ919-4402-0062, and China Postdoctoral Science Foundation, project nos. 2020M673218 and 2020TQ0222.

\section{References}

[1] O. Schmidt, A. Hawkes, A. Gambhir, and I. Staffell, "The future cost of electrical energy storage based on experience rates," Nature Energy, vol. 2, no. 8, Article ID 17110, 2017.
[2] F. Feng, X. Hu, L. Hu, F. Hu, Y. Li, and L. Zhang, "Propagation mechanisms and diagnosis of parameter inconsistency within Li-Ion battery packs," Renewable and Sustainable Energy Reviews, vol. 112, pp. 102-113, 2019.

[3] F. Feng, X. Hu, J. Liu, X. Lin, and B. Liu, "A review of equalization strategies for series battery packs: variables, objectives, and algorithms," Renewable and Sustainable Energy Reviews, vol. 116, Article ID 109464, 2019.

[4] K. Liu, Y. Shang, Q. Ouyang, and W. D. Widanage, "A datadriven approach with uncertainty quantification for predicting future capacities and remaining useful life of lithiumion battery," IEEE Transactions on Industrial Electronics, vol. 2020, p. 1, 2020.

[5] J. Meng, M. Ricco, G. Luo et al., "An overview and comparison of online implementable SOC estimation methods for lithium-ion battery," IEEE Transactions on Industry Applications, vol. 54, no. 2, pp. 1583-1591, 2018.

[6] K. Liu, K. Li, Z. Yang, C. Zhang, and J. Deng, "Battery optimal charging strategy based on a coupled thermoelectric model," in Proceedings of the IEEE Congress on Evolutionary Computation (CEC), pp. 5084-5091, Vancouver, Canada, July 2016.

[7] D. I. Stroe, V. Knap, and E. Schaltz, "State-of-health estimation of lithium-ion batteries based on partial charging voltage profiles," ECS Transactions, vol. 85, no. 13, pp. 379-386, 2018.

[8] H. Dai, G. Zhao, M. Lin, J. Wu, and G. Zheng, "A novel estimation method for the state of health of lithium-ion battery using prior knowledge-based neural network and Markov chain," IEEE Transactions on Industrial Electronics, vol. 66, no. 10, pp. 7706-7716, 2019.

[9] F. Feng, X. Hu, K. Liu et al., "A practical and comprehensive evaluation method for series-connected battery pack models," IEEE Transactions on Transportation Electrification, vol. 6, no. 2, pp. 391-416, 2020.

[10] L. Lu, X. Han, J. Li, J. Hua, and M. Ouyang, "A review on the key issues for lithium-ion battery management in electric vehicles," Journal of Power Sources, vol. 226, pp. 272-288, 2013.

[11] C. S. Huang, T. W.-S. Chow, and M.-Y. Chow, "Li-ion battery parameter identification with low pass filter for measurement noise rejection," in Proceeding of the 2017 IEEE 26th International Symposium on Industrial Electronics (ISIE), pp. 2075-2080, Delft, The Netherlands, June 2017.

[12] Q. Ouyang, Z. Wang, K. Liu, G. Xu, and Y. Li, "Optimal charging control for lithium-ion battery packs: a distributed average tracking approach," IEEE Transactions on Industrial Informatics, vol. 16, no. 5, pp. 3430-3438, 2020.

[13] X. Tang, K. Liu, X. Wang, F. Gao, J. Macro, and W. D. Widanage, "Model migration neural network for predicting battery aging trajectories," IEEE Transactions on Transportation Electrification, vol. 6, no. 2, pp. 363-374, 2020.

[14] S. M. Lukic, J. Jian Cao, R. C. Bansal, F. Rodriguez, and A. Emadi, "Energy storage systems for automotive applications," IEEE Transactions on Industrial Electronics, vol. 55, no. 6, pp. 2258-2267, 2008.

[15] K. Liu, Y. Li, X. Hu, M. Lucu, and W. D. Widanage, "Gaussian process regression with automatic relevance determination 
kernel for calendar aging prediction of lithium-ion batteries," IEEE Transactions on Industrial Informatics, vol. 2020, 2020.

[16] K. Liu, X. Hu, Z. Wei, Y. Li, and Y. Jiang, "Modified Gaussian process regression models for cyclic capacity prediction of lithium-ion batteries," IEEE Transactions on Transportation Electrification, vol. 2019, 2019.

[17] Z. Chen, Y. Fu, and C. C. Mi, "State of charge estimation of lithium-ion batteries in electric drive vehicles using extended kalman filtering," IEEE Transactions on Vehicular Technology, vol. 62, no. 3, pp. 1020-1030, 2013.

[18] M. Cacciato, G. Nobile, G. Scarcella, and G. Scelba, "Real-time model-based estimation of SOC and SOH for energy storage systems," IEEE Transactions on Power Electronics, vol. 32, no. 1, pp. 794-803, 2017.

[19] X. Tang, K. Liu, X. Wang, B. Liu, F. Gao, and W. D. Widanage, "Real-time aging trajectory prediction using a base modeloriented gradient-correction particle filter for Lithium-ion batteries," Journal of Power Sources, vol. 440, Article ID 227118, 2019.

[20] L. Cai, J. Meng, D.-I. Stroe, J. Peng, G. Luo, and R. Teodorescu, "Multiobjective optimization of data-driven model for lithium-ion battery SOH estimation with short-term feature," IEEE Transactions on Power Electronics, vol. 35, no. 11, pp. 11855-11864, 2020.

[21] B. Balagopal and M. Y. Chow, "The state of the art approaches to estimate the state of health $(\mathrm{SOH})$ and state of function (SOF) of lithium Ion batteries," in Proceeding of the-2015 IEEE International Conference on Industrial Informatics INDIN, pp. 1302-1307, Cambridge, UK, July 2015.

[22] M. Berecibar, I. Gandiaga, I. Villarreal, N. Omar, J. Van Mierlo, and P. Van Den Bossche, "Critical review of state of health estimation methods of Li-ion batteries for real applications," Renewable and Sustainable Energy Reviews, vol. 56, pp. 572-587, 2016.

[23] K. Liu, T. R. Ashwin, X. Hu, M. Lucu, and W. D. Widanage, "An evaluation study of different modelling techniques for calendar ageing prediction of lithium-ion batteries," Renewable and Sustainable Energy Reviews, vol. 131, Article ID 110017, 2020.

[24] Y. Xing, J. Na, and R. Costa-Castelló, "Real-time adaptive parameter estimation for a polymer electrolyte membrane fuel cell," IEEE Transactions on Industrial Informatics, vol. 2019, 2019.

[25] J. Meng, G. Luo, M. Ricco, M. Swierczynski, D.-I. Stroe, and R. Teodorescu, "Overview of lithium-Ion battery modeling methods for state-of-charge estimation in electrical vehicles," Applied Sciences, vol. 8, no. 5, p. 659, 2018.

[26] F. Feng, S. Teng, K. Liu et al., "Co-estimation of lithium-ion battery state of charge and state of temperature based on a hybrid electrochemical-thermal-neural-network model," Journal of Power Sources, vol. 455, Article ID 227935, 2020.

[27] J. Na, Y. Xing, and R. Costa-Castelló, "Adaptive estimation of time-varying parameters with application to roto-magnet plant," IEEE Transactions on Systems, Man, and Cybernetics: Systems, vol. 2018, pp. 1-11, 2018.

[28] L. Zhou, Y. Zheng, M. Ouyang, and L. Lu, "A study on parameter variation effects on battery packs for electric vehicles," Journal of Power Sources, vol. 364, pp. 242-252, 2017.

[29] B. Duan, Z. Li, P. Gu, Z. Zhou, and C. Zhang, "Evaluation of battery inconsistency based on information entropy," Journal of Energy Storage, vol. 16, pp. 160-166, 2018.

[30] J. Zhang, Y. Wei, and H. Qi, "State of charge estimation of $\mathrm{LiFePO} 4$ batteries based on online parameter identification," Applied Mathematical Modelling, vol. 40, no. 11-12, pp. 6040-6050, 2016.
[31] Q. Yu, R. Xiong, C. Lin, W. Shen, and J. Deng, "Lithium-ion battery parameters and state-of-charge joint estimation based on $\mathrm{H}$-infinity and unscented kalman filters," IEEE Transactions on Vehicular Technology, vol. 66, no. 10, pp. 8693-8701, 2017.

[32] J. Meng, D.-I. Stroe, M. Ricco, G. Luo, and R. Teodorescu, “A simplified model-based state-of-charge estimation approach for lithium-ion battery with dynamic linear model," IEEE Transactions on Industrial Electronics, vol. 66, no. 10, pp. 7717-7727, 2019.

[33] G. L. Plett, "Extended kalman filtering for battery management systems of LiPB-based HEV battery packs," Journal of Power Sources, vol. 134, no. 2, pp. 277-292, 2004.

[34] H. Dai, T. Xu, L. Zhu, X. Wei, and Z. Sun, "Adaptive model parameter identification for large capacity Li-ion batteries on separated time scales," Applied Energy, vol. 184, pp. 119-131, 2016.

[35] H. Rahimi-Eichi, F. Baronti, and M.-Y. Chow, "Online adaptive parameter identification and state-of-charge coestimation for lithium-polymer battery cells," IEEE Transactions on Industrial Electronics, vol. 61, no. 4, pp. 2053-2061, 2014.

[36] J. Meng, M. Ricco, A. B. Acharya et al., "Low-complexity online estimation for LiFePO4 battery state of charge in electric vehicles," Journal of Power Sources, vol. 395, pp. 280-288, 2018.

[37] C. Zhang, W. Allafi, Q. Dinh, P. Ascencio, and J. Marco, "Online estimation of battery equivalent circuit model parameters and state of charge using decoupled least squares technique," Energy, vol. 142, pp. 678-688, 2018.

[38] H. He, R. Xiong, X. Zhang, F. Sun, and J. Fan, "State-of-charge estimation of the lithium-ion battery using an adaptive extended Kalman filter based on an improved Thevenin model," IEEE Transactions on Vehicular Technology, vol. 60, no. 4, pp. 1461-1469, 2011.

[39] L. Gregory, "Sigma-point kalman filtering for battery management systems of LiPB-based HEV battery packs part 1: introduction and state estimation," Journal of Power Sources, vol. 161, no. 2, 2006.

[40] L. Chen, "A novel state-of-charge estimation method of lithium-ion batteries combining the grey model and genetic algorithms," IEEE Transactions on Power Electronics, vol. 8993, 2017.

[41] Z. Yu, L. Xiao, H. Li, X. Zhu, and R. Huai, "Model parameter identification for lithium batteries using the coevolutionary particle Swarm optimization method," IEEE Transactions on Industrial Electronics, vol. 64, no. 7, pp. 5690-5700, 2017.

[42] P. C. Young, "Refined instrumental variable estimation: maximum likelihood optimization of a unified box-jenkins model," Automatica, vol. 52, pp. 35-46, 2015.

[43] X. Tang, Y. Wang, C. Zou, K. Yao, Y. Xia, and F. Gao, “A novel framework for lithium-ion battery modeling considering uncertainties of temperature and aging," Energy Conversion and Management, vol. 180, pp. 162-170, 2019.

[44] X. Hu, S. Li, and H. Peng, "A comparative study of equivalent circuit models for Li-ion batteries," Journal of Power Sources, vol. 198, pp. 359-367, 2012.

[45] S. Nejad, D. T. Gladwin, and D. A. Stone, "A systematic review of lumped-parameter equivalent circuit models for real-time estimation of lithium-ion battery states," Journal of Power Sources, vol. 316, pp. 183-196, 2016.

[46] P. Young, "Some observations on instrumental variable methods of time-series analysis," International Journal of Control, vol. 23, no. 5, pp. 593-612, 1976.

[47] P. C. Young, Recursive Least Squares Estimation Recursive Estimation And Time-Series Analysis, pp. 29-46, Springer, Berlin, Germany, 2011. 
[48] X. Tang, C. Zou, T. Wik et al., "Run-to-Run control for active balancing of lithium iron phosphate battery packs," IEEE Transactions on Power Electronics, vol. 35, no. 2, pp. 14991512, 2020.

[49] P. Malysz, J. Ye, R. Gu, H. Yang, and A. Emadi, "Battery stateof-power peak current calculation and verification using an asymmetric parameter equivalent circuit model," IEEE Transactions on Vehicular Technology, vol. 65, no. 6, pp. 4512-4522, 2016. 